SiRNAs and innate immunity

\title{
Innate Immunity confounds the clinical efficacy of small interfering RNAs (siRNAs)
}

JJ Rossi

Gene Therapy (2009) 16, 579-580; doi:10.1038/gt.2009.26; published online 5 March 2009

Small interfering RNAs (siRNAs) are double stranded, sequence-specific inhibitors of gene expression. They act by incorporation into the catalytic domain of an Argonaute family protein serving as guides for a complex that functionally destroys the target. The discovery that they can trigger site-specific cleavage in the absence of type I interferon induction. ${ }^{1}$ has led to a plethora of inhibitory applications for these small duplexes in mammalian systems. However, several recent findings demonstrate that, under appropriate conditions, siRNAs can trigger interferon responses. This has confounded interpretations of the role sequence-specific inhibition plays in observed siRNA effects. ${ }^{2-5}$

RNA interference is one of the most important recent discoveries in cell biology. ${ }^{6}$ The rapid progression from discovery to applications is unprecedented, and there are currently numerous human clinical trials using siRNAs to treat diseases from macular degeneration to HIV. 7 Additionally, there are a number of biotech start-up companies that have siRNA therapeutics as a primary focus. An important selling point is the sequence specificity in gene inhibition, but this feature was challenged in 2006 when Jackson et al. ${ }^{8}$ revealed siRNAs binding to nontargeted areas can trigger off-target inhibition of gene expression. Fortunately, a minor modification of a single 2' O-methyl group at the second ribose from the $5^{\prime}$ end of each RNA strand was shown to abrogate this problem.

A second setback for therapeutic application, however, occurred when it was demonstrated that certain siRNA sequence motifs activate toll-like receptors (TLRs- Figure 1), which induced interferon and, therefore, a non-sequence-specific downregulation of gene expression. These published observations once again raised concerns that siRNAs might not be safe for clinical applications. ${ }^{2,3}$ A solution for the problem again involved simple 2' O-methyl modifications judiciously placed on guanosine and uridine backbones along the siRNAs, which prevented cytoplasmic TLR activation and type I interferon induction. ${ }^{9}$ Unfortunately, several siRNA clinical trials were initiated either before, or without consideration of, these findings, which caused concerns about the efficacy of these molecules.

One of these trials was for adult late stage wet macular degeneration (AMD), a disease characterized by neo-vascularization of the macula in the eye, ultimately resulting in blindness. This disease was the first target for siRNA therapy. Several companies have developed a treatment involving intraocular injection of siRNAs targeting either Vegf or the Vegf receptor to block neo-vascularization in the eye. ${ }^{10} \mathrm{~A}$ recent study using rodent models, however, revealed an unexpected observation that neo-vascularization of the macula could be prevented by a variety of siRNAs, and was not specific for anti-vegf or vegf receptor siRNAs. ${ }^{4}$ In these studies it was also observed that siRNAs need not be directly injected into the eye, but could be injected into the peritoneum of animals and yield the same results. Further investigations revealed the siRNAs were interacting with the double-stranded RNA receptor TLR3 (Figure 1), which in turn triggered upregulation of gamma interferon and interleukin 12, setting off a cascade of events that downregulated the neovascular processes, including downregulation of expression of the vegf message. Importantly, in this study, the use of naked RNAs with a $2^{\prime}$ O-methyl modification still activated TLR3, thus setting this pathway apart from the other TLRs. This stunning set of results from well controlled experiments has left investigators wondering how many of the pre-clinical efficacious results of siRNA applications are not due to the specific message targeting, but can be explained by innate immune responses to doublestranded siRNAs.

This line of investigation was taken further by a recent study which questions the use of siRNAs for treating influenza infection in a murine model. ${ }^{5}$ Using published siRNAs targeting murine influenza transcripts, Robbins et al., tested the inhibitory effects of unmodified versus $2^{\prime} \mathrm{O}$-methyl-modified siRNAs in cell culture, and showed that both types of siRNAs were inhibitory to viral infection. When these same siRNAs were encapsulated in a PEIlipid complex and infused into the lungs, only the non-modified siRNA had an antiviral effect. ${ }^{5}$ They went on to demonstrate that an unmodified anti-ApoB siRNA also had antiviral activity in vivo. From these results, the investigators reasoned that the unmodified siRNAs were triggering a TLR response and type I interferon induction which was responsible for the antiviral effect while siRNAs with a modified backbone did not trigger this pathway. Measurements of type I interferon induction confirmed this. Finally, the researchers showed a commonly employed antiEGFP siRNA used as a negative control in previously published influenza studies lacked this immunostimulatory effect. The reason why this sequence is not immunostimulatory, when most other siRNAs are, is unclear at this time but importantly this EGFP siRNA has been used as a negative control in 15 previously published studies, bringing into question the validity of the results from these studies.

What are the implications of the Robbins et al., ${ }^{5}$ and also the Kleinman et al., ${ }^{4}$ studies for siRNA therapeutics? At this time it is not entirely clear whether or not the efficacies observed in early clinical trials using unmodified siRNAs are due to TLR activation, are solely due to siRNA mediated target down regulation, or are a combination of both. These studies point to the importance of understanding the mechanisms of TLR activation; TLR3 is a cell surface receptor while TLR 7 and 8 are 


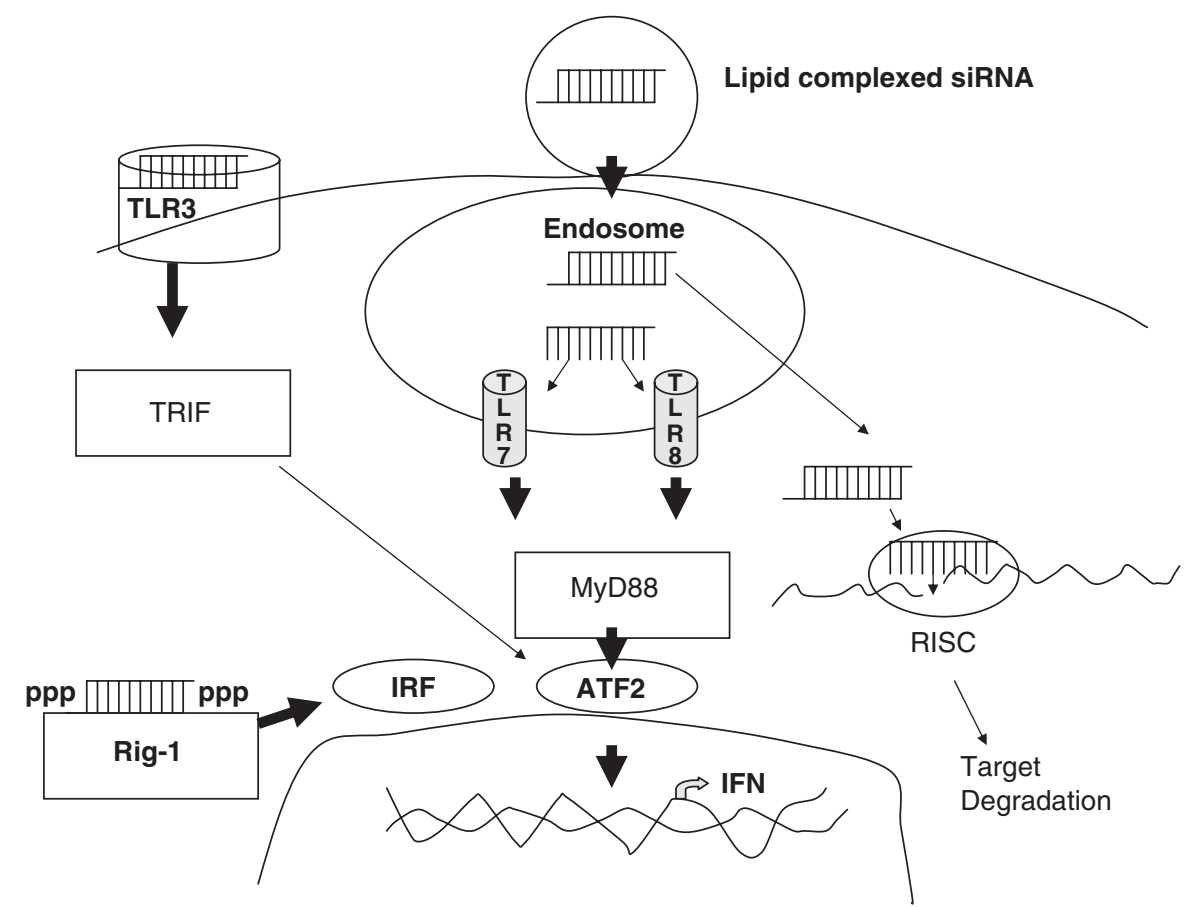

Figure 1 SiRNA interactions with toll-like receptors and the RNA interference gene silencing pathway. The various toll-like receptors are depicted. TLR7 and TLR8 recognize ssRNA motifs, but may also recognize certain motifs in dsRNAs. TLR3 binds dsRNA and signals through a different pathway from TLR7 and 8. RIG-1 recognizes triphosphate containing RNAs. The siRNAs that escape from the endosome can enter the RNAi pathway where one of the two strands is selected as a guide strand and directs sequence-specific message degradation of a complementary RNA.

cytoplasmic and all of these can potentially be activated by siRNAs. What is clear is that activation of the intracellular TLRs can be avoided by the simple 2' O-methyl backbone modification reported by Judge et al. ${ }^{11}$ This modification along with the use of a delivery vehicle to avoid the cell surface receptor TLR3 are safety measures that should be employed in any future siRNA clinical trials. Importantly, the EGFP siRNA control which lacks immunostimulatory activity ${ }^{5}$ should also be avoided as a control for in vivo experiments or clinical trials.

In considering the take-home message from these findings, it may be advantageous to look at combining siRNA mediated, target-specific downregulation with siRNA-induced stimulation of the interferon pathway. A recently published study has shown that tri-phosphate-containing siRNAs, which are potent triggers of type I interferon induction, resulted in potent inhibition of metastatic melanoma tumor progression in a murine model. ${ }^{12}$ For treatment of other conditions though, the dual effect may not be beneficial, and only the sequence specificity of the siRNA would be useful. It will require judicious testing of backbone modifications and delivery mechanisms in vivo to ensure siRNA applications are only targeting the desired transcripts so specific gene downregulation is the sole cause of observed phenotypes.

Dr J Rossi is at Department of, Molecular Biology, Beckman Research Institute, Beckman Research Institute of the City of Hope, 1450 E. Duarte Rd., Duarte, CA, 91010, USA.

E-mail: jrossi@coh.org

1 Elbashir SM, Harborth J, Lendeckel W, Yalcin A, Weber K, Tuschl T. Duplexes of 21-nucleotide RNAs mediate RNA interference in cultured mammalian cells. Nature 2001; 411: 494-498.

2 Hornung V, Guenthner-Biller $\mathrm{M}$, Bourquin C, Ablasser A, Schlee M, Uematsu $S$ et al. Sequence-specific potent induction of IFN-alpha by short interfering RNA in plasmacytoid dendritic cells through TLR7. Nature Medicine 2005; 11: 263-270.

3 Judge $\mathrm{AD}$, Sood V, Shaw JR, Fang D, McClintock K, MacLachlan I. Sequencedependent stimulation of the mammalian innate immune response by synthetic siRNA. Nature Biotechnology 2005; 23: 457-462.

4 Kleinman ME, Yamada K, Takeda A, Chandrasekaran V, Nozaki M, Baffi JZ et al. Sequence- and target-independent angiogenesis suppression by siRNA via TLR3. Nature 2008; 452: 591-597.
5 Robbins M, Judge A, Ambegia E, Choi C, Yaworski E, Palmer L et al. Misinterpreting the therapeutic effects of siRNA caused by immune stimulation. Human Gene Therapy 2008 (in press).

6 Fire A, Xu S, Montgomery MK, Kostas SA, Driver SE, Mello CC. Potent and specific genetic interference by doublestranded RNA in Caenorhabditis elegans. Nature 1998; 391: 806-811.

$7 \mathrm{Kim}$ DH, Rossi JJ. Strategies for silencing human disease using RNA interference. Nature Reviews Genetics 2007; 8: 173-184.

8 Jackson AL, Burchard J, Leake D, Reynolds A, Schelter J, Guo J et al. Position-specific chemical modification of siRNAs reduces "off-target" transcript silencing. Rna 2006; 12: 1197-1205.

9 Robbins M, Judge A, Liang L, McClintock $\mathrm{K}$, Yaworski E, MacLachlan I. 2'-Omethyl-modified RNAs act as TLR7 antagonists. Mol Ther 2007; 15: 1663-1669.

10 Michels S, Schmidt-Erfurth U, Rosenfeld PJ. Promising new treatments for neovascular age-related macular degeneration. Expert Opinion On Investigational Drugs 2006; 15: 779-793.

11 Judge AD, Bola G, Lee AC, MacLachlan I. Design of noninflammatory synthetic siRNA mediating potent gene silencing in vivo. Mol Ther 2006; 13: 494-505.

12 Poeck H, Besch R, Maihoefer C, Renn M, Tormo D, Morskaya SS et al. 5'-Triphosphate-siRNA: turning gene silencing and Rig-I activation against melanoma. Nature Medicine 2008; 14: 1256-1263. 